\title{
On the Riesz means of $\delta_{k}(n)$
}

\author{
Saurabh Kumar Singh
}

\begin{abstract}
Let $k \geq 1$ be an integer. Let $\delta_{k}(n)$ denote the maximum divisor of $n$ which is co-prime to $k$. We study the error term of the general $m$-th Riesz mean of the arithmetical function $\delta_{k}(n)$ for any positive integer $m \geq 1$, namely the error term $E_{m, k}(x)$ where

$$
\frac{1}{m !} \sum_{n \leq x} \delta_{k}(n)\left(1-\frac{n}{x}\right)^{m}=M_{m, k}(x)+E_{m, k}(x) .
$$
\end{abstract}

We establish a non-trivial upper bound for $\left|E_{m, k}(x)\right|$, for any integer $m \geq 1$.

Keywords. Euler-totient function, Generating functions, Riemann zeta-function.

2010 Mathematics Subject Classification. Primary 11A25; Secondary 11N37.

\section{Introduction}

For any fixed positive integer $k$, we define

$$
\delta_{k}(n)=\max \{d: d \mid n, \quad(d, k)=1\} .
$$

Joshi and Vaidya $[J V]$ proved that

$$
\sum_{n \leq x} \delta_{k}(n)=\frac{k}{2 \sigma(k)} x^{2}+E_{k}(x),
$$

with $E_{k}(x)=O(x)$ and $\sigma(k)=\sum_{d \mid k} d$, when $k$ is a square free positive integer. They also proved that when $k=p$, a prime,

$$
\varliminf_{n \rightarrow \infty} \frac{E_{p}(x)}{x}=-\frac{p}{p+1}, \quad \text { and } \quad \varlimsup_{n \rightarrow \infty} \frac{E_{p}(x)}{x}=\frac{p}{p+1} .
$$

It was proved by Maxsein and Herzog $[\mathrm{MH}]$ that for any square free positive integer $k$,

$$
\varliminf_{n \rightarrow \infty} \frac{E_{k}(x)}{x} \leq-\frac{k}{\sigma(k)}, \quad \text { and } \quad \varlimsup_{n \rightarrow \infty} \frac{E_{k}(x)}{x} \geq \frac{k}{\sigma(k)}
$$

Around the same time, Adhikari, Balasubramanian and Sankaranarayanan [ABS] proved the above results by a different method. While a tauberian theorem of Hardy-Littlewood and Karamata was used in $[\mathrm{MH}]$ to get the asymptotic formula for $\sum_{n \leq x} \gamma_{k}(n)$, where $\gamma_{k}(n)$ is defined by the relation $\delta_{k}(n)=\gamma_{k} * I(n)$ where $*$ is the Dirichlet convolution and $I$ is the identity function, the method of [ABS] consists of averaging over arithmetical progressions.

For $k \geq 1$ and square free, Harzog and Maxsein [MH] had also observed that

$$
\limsup _{x \rightarrow \infty} \frac{E_{k}(x)}{x} \leq \frac{1}{2} d(k)
$$

We thank episciences.org for providing open access hosting of the electronic journal Hardy-Ramanujan Journal 
where $d(k)$ denotes the number of divisors of $k$. Later Adhikari and Balasubramanian [AB] improved this result of Maxsein and Herzog by showing that

$$
\varlimsup_{n \rightarrow \infty} \frac{\left|E_{k}(x)\right|}{x} \leq \frac{1}{2}\left(1-\frac{1}{p+1}\right) d(k)
$$

where $p$ denotes the smallest prime dividing $k$.

Writing

$$
H_{k}(x)=\sum_{n \leq x} \frac{\delta_{k}(n)}{n}-\frac{k x}{\sigma(k)}
$$

one observes (see $[\mathrm{ABS}])$ that

$$
\frac{E_{k}(x)}{x}=H_{k}(x)+O(1) .
$$

In [AS], more precise upper and lower bounds for the quantities $\underline{\lim } H_{k}(x)$ and $\varlimsup H_{k}(x)$ were established. The aim of this article is to study the error term of the general $m$-th Riesz mean related to the arithmetic function $\delta_{k}(n)$ for any positive integer $m \geq 1$ and $k \geq 1$. More precisely, we write

$$
\frac{1}{m !} \sum_{n \leq x} \delta_{k}(n)\left(1-\frac{n}{x}\right)^{m}=M_{m, k}(x)+E_{m, k}(x)
$$

where $M_{m, k}(x)$ is the main term (exists) and $E_{m, k}(x)$ is the error term of the sum under investigation. We prove the following.

Theorem 1.1. Let $x \geq x_{0}$ where $x_{0}$ is a sufficiently large positive number and let $c(\eta)=\frac{2}{1-2^{-\eta}}$ for any $\eta>0$. For any integer $m \geq 1$ and for any integer $k \geq 1$, we have

$$
\frac{1}{m !} \sum_{n \leq x} \delta_{k}(n)\left(1-\frac{n}{x}\right)^{m}=\frac{x^{2}}{(m+2) !} \prod_{p \mid k} \frac{p}{p+1}+E_{m, k}(x),
$$

where

$$
E_{1, k}(x) \ll k c(1 / 2)^{\omega(k)} x^{\frac{1}{2}} \log x
$$

and for $m \geq 2$, we have

$$
E_{m, k}(x) \ll k c(\eta)^{\omega(k)} x^{\eta}
$$

for any small fixed positive constant $\eta$ and the implied constant is independent of $m$.

\section{Notation}

1. Throughout the paper, $s=\sigma+i t$; the parameters $T$ and $x$ are sufficiently large real numbers and $m$ is an integer $\geq 1$.

2. $\eta, \epsilon$ always denote sufficiently small positive constants.

3. As usual $\zeta(s)$ denotes the Riemann zeta-function.

4. $k$ is any square free positive integer. 


\section{Some Lemmas}

Generating function for $\delta_{k}(n)$ is given by:

Lemma 3.1. We have

$$
\sum_{n=1}^{\infty} \frac{\delta_{k}(n)}{n^{s}}=\zeta(s-1) G(s)
$$

where

$$
G(s)=\sum_{n=1}^{\infty} \frac{g(n)}{n^{s}}=\prod_{p \mid k}\left(\frac{1-\frac{p}{p^{s}}}{1-\frac{1}{p^{s}}}\right) \ll k c(\eta)^{\omega(k)}
$$

for $\sigma \geq \eta$ and

$$
c(\eta)=\frac{2}{1-2^{-\eta}} .
$$

Proof. We have (see [ABS, equation 2.2]),

$$
\begin{aligned}
\sum_{n=2}^{\infty} \frac{\delta_{k}(n)}{n^{s}} & =\prod_{p}\left(1+\frac{\delta_{k}(p)}{p^{s}}+\frac{\delta_{k}\left(p^{2}\right)}{p^{2 s}}+\cdots\right) \\
& =\prod_{p \mid k}\left(1+\frac{1}{p^{s}}+\frac{1}{p^{2 s}}+\cdots\right) \prod_{p \nmid k}\left(1+\frac{p}{p^{s}}+\frac{p^{2}}{p^{2 s}}+\cdots\right) \\
& =\zeta(s-1) \prod_{p \mid k} \frac{1-\frac{1}{p^{s-1}}}{1-\frac{1}{p^{s}}}:=\zeta(s-1) G(s)
\end{aligned}
$$

since

$$
\delta_{k}\left(p^{m}\right)=\left\{\begin{array}{lll}
1 & \text { if } & p \mid k \\
p^{m} & \text { if } & p \nmid k .
\end{array}\right.
$$

And for $\sigma \geq \eta(>0)$, we observe that

$$
|G(s)|=\prod_{p \mid k}\left|\frac{1-\frac{1}{p^{s-1}}}{1-\frac{1}{p^{s}}}\right| \leq \prod_{p \mid k} \frac{1+p^{1-\eta}}{1-\frac{1}{p^{\eta}}} \leq \prod_{p \mid k} \frac{2 p}{1-\frac{1}{2^{\eta}}} \leq k c(\eta)^{\omega(k)} .
$$

Lemma 3.2. Let $m$ be an integer $\geq 1$. Let $c$ and $y$ be any positive real numbers and $T \geq T_{0}$ where $T_{0}$ is sufficiently large. Then we have,

$$
\frac{1}{2 \pi i} \int_{c-i T}^{c+i T} \frac{y^{s}}{s(s+1) \cdots(s+m)} d s= \begin{cases}\frac{1}{m !}\left(1-\frac{1}{y}\right)^{m}+O\left(\frac{4^{m} y^{c}}{T^{m}}\right) & \text { if } y \geq 1 \\ O\left(\frac{1}{T^{m}}\right) & \text { if } 0<y \leq 1\end{cases}
$$

Proof. See [SS, Lemma 3.2] and also [In, p.31 Theorem B]).

Lemma 3.3. The Riemann zeta-function $\zeta(s)$ is extended as a meromorphic function in the whole complex plane $\mathbb{C}$ with a simple pole at $s=1$ and it satisfies a functional equation $\zeta(s)=\chi(s) \zeta(1-s)$ where

$$
\chi(s)=\frac{\pi^{-(1-s) / 2} \Gamma\left(\frac{1-s}{2}\right)}{\pi^{-s / 2} \Gamma\left(\frac{s}{2}\right)} .
$$

Also, in any bounded vertical strip, using Stirling's formula, we have 


$$
\chi(s)=\left(\frac{2 \pi}{t}\right)^{\sigma+i t-1 / 2} e^{i\left(t+\frac{\pi}{4}\right)}\left(1+O\left(t^{-1}\right)\right)
$$

as $|t| \rightarrow \infty$. Thus, in any bounded vertical strip,

$$
|\chi(s)| \asymp t^{1 / 2-\sigma}\left(1+O\left(t^{-1}\right)\right)
$$

as $|t| \rightarrow \infty$.

Proof. See [T, p.116] or [Iv, p.8-12].

Lemma 3.4. We have for $t \geq t_{0}$ (sufficiently large),

$$
\zeta\left(\frac{1}{2}+i t\right) \ll t^{1 / 6}(\log t)^{3 / 2}
$$

and

$$
\zeta(1+i t) \ll \log t
$$

Proof. See [T, page 99, Theorem 5.5] and [T, page 49, Theorem 3.5]

\section{Proof of theorem 1.1}

From Lemma 3.2, with $c=2+\frac{1}{\log x}$ and writing $F(s):=\zeta(s-1) G(s)$, we have

$$
\begin{aligned}
S:=\sum_{n \leq x} \delta_{k}(n)\left(1-\frac{n}{x}\right)^{m} & =\frac{1}{2 \pi i} \int_{c-i \infty}^{c+i \infty} F(s) \frac{x^{s}}{s(s+1) \cdots(s+m)} d s \\
& =\frac{1}{2 \pi i} \int_{c-i T}^{c+i T} F(s) \frac{x^{s}}{s(s+1) \cdots(s+m)} d s+O\left(\frac{4^{m} x^{c} \log x}{T^{m}}\right) .
\end{aligned}
$$

Note that the tail portion error term in the above expression is actually

$$
\ll \frac{4^{m}}{T^{m}} x^{c} \sum_{n \leq x} \frac{\delta_{k}(n)}{n^{c}} \ll \frac{4^{m} x^{c} \log x}{T^{m}},
$$

since $\delta_{k}(n) \leq n$.

Case 1: Let $m=1$. We move the line of integration in the above integral to $\Re s=\frac{1}{2}$. In the rectangular contour formed by the line segments joining the points $c-i T, c+i T, \frac{1}{2}+i T, \frac{1}{2}-i T$ and $c-i T$ in the anticlockwise order, we observe that $s=2$ is a simple pole of the integrand. Thus we get the main term $\frac{x^{2}}{(m+2) !} \prod_{p \mid k} \frac{p}{p+1}$ from the residue coming from the pole $s=2$.

We note that

$$
\begin{aligned}
& \frac{1}{2 \pi i} \int_{c-i T}^{c+i T} F(s) \frac{x^{s}}{s(s+1)} d s \\
& =\frac{1}{2 \pi i}\left\{\int_{\frac{1}{2}+i T}^{c+i T} \cdots+\int_{\frac{1}{2}-i T}^{\frac{1}{2}+i T} \cdots+\int_{c-i T}^{\frac{1}{2}-i T} \cdots\right\}+\text { sum of the residues. }
\end{aligned}
$$

The left vertical line segment contributes the quantity: 


$$
\begin{aligned}
Q_{1} & :=\frac{1}{2 \pi} \int_{-T}^{T} F(1 / 2+i t) \frac{x^{1 / 2+i t} d t}{(-1 / 2+i t)(1 / 2+i t)} d t \\
& =\frac{1}{2 \pi}\left(\int_{t \mid \leq t_{0}}+\int_{t_{0}<|t| \leq T}\right) \frac{x^{\frac{1}{2}+i t} \zeta\left(-\frac{1}{2}+i t\right) G\left(\frac{1}{2}+i t\right) d t}{\left.\left(\frac{1}{2}+i t\right)\left(\frac{1}{2}+i t\right)\right)} \\
& \ll k c(1 / 2)^{\omega(k)} x^{1 / 2}+k c(1 / 2)^{\omega(k)} x^{1 / 2} \int_{t_{0}<|t| \leq T} t^{1 / 2-(-1 / 2)}\left|\zeta(3 / 2+i t) G\left(\frac{1}{2}+i t\right)\right| \frac{d t}{t^{2}} \\
& \ll k c(1 / 2)^{\omega(k)} x^{1 / 2}+k c(1 / 2)^{\omega(k)} x^{1 / 2} \int_{t_{0}<t \leq T} \frac{d t}{t} . \\
& \ll k c(1 / 2)^{\omega(k)} x^{1 / 2} \log T .
\end{aligned}
$$

Now we will estimate the contributions coming from the upper horizontal line (estimation for the lower horizontal line is similar).

The horizontal lines in total contribute a quantity which is in absolute value

$$
\begin{aligned}
& \ll \int_{1 / 2}^{c}\left|\zeta(\sigma-1+i T) G(\sigma+i T) \frac{x^{\sigma+i T}}{(\sigma+i T)(\sigma+1+i T)}\right| d \sigma \\
& \ll\left(\int_{1 / 2}^{1}+\int_{1}^{3 / 2}+\int_{3 / 2}^{c}\right)|\zeta(\sigma-1+i T) G(\sigma+i T)| \frac{x^{\sigma}}{T^{2}} d \sigma \\
& \ll k c(1 / 2)^{\omega(k)}\left\{\left(\int_{1 / 2}^{1}+\int_{1}^{3 / 2}\right) T^{1 / 2-\sigma+1}|\zeta(2-\sigma+i T)| \frac{x^{\sigma}}{T^{2}} d \sigma\right. \\
& \left.+\int_{3 / 2}^{c}|\zeta(\sigma-1+i T)| \frac{x^{\sigma}}{T^{2}} d \sigma\right\}(\text { by Lemma 3.3) } \\
& \ll k c(1 / 2)^{\omega(k)}\left(\frac{x \log T}{T}+\frac{x^{3 / 2} \log T}{T^{3 / 2}}+\frac{x^{2} \log T}{T^{11 / 6}}\right) \text { (by Lemma 3.4). }
\end{aligned}
$$

Collecting all the estimates, and taking $T=x^{10}$ we get:

$$
\begin{aligned}
E_{1, k}(x) & \ll k c(1 / 2)^{\omega(k)}\left(x^{1 / 2} \log T+\frac{x^{2}}{T}+\frac{x \log T}{T}+\frac{x^{3 / 2} \log T}{T^{3 / 2}}+\frac{x^{2} \log T}{T^{11 / 6}}\right) \\
& \ll k c(1 / 2)^{\omega(k)} x^{1 / 2} \log x .
\end{aligned}
$$

Case 2: Let $m \geq 2$. We move the line of integration to $\Re s=\eta(>0)$.

We note that

$$
\begin{aligned}
& \frac{1}{2 \pi i} \int_{c-i T}^{c+i T} F(s) \frac{x^{s}}{s(s+1) \cdots(s+m)} d s \\
& =\frac{1}{2 \pi i}\left\{\int_{\delta+i T}^{c+i T} \cdots+\int_{\delta-i T}^{\delta+i T} \cdots+\int_{c-i T}^{\delta-i T} \cdots\right\}+\text { sum of the residue. }
\end{aligned}
$$

The left vertical line segment contributes the quantity: 


$$
\begin{aligned}
Q_{m} & :=\frac{1}{2 \pi} \int_{-T}^{T} F(\eta+i t) \frac{x^{\eta+i t} d t}{(\eta+i t)(\eta+1+i t) \cdots(\eta+m+i t)} d t \\
& =\frac{1}{2 \pi}\left(\int_{t \mid \leq t_{0}}+\int_{t_{0}<|t| \leq T}\right) \frac{x^{\eta+i t} \zeta(\eta-1+i t) G(\eta+i t) d t}{(\eta+i t)(\eta+1+i t) \cdots(\eta+m+i t)} \\
& \ll k c(\eta)^{\omega(k)} x^{\eta}+k c(\eta)^{\omega(k)} x^{\eta} \int_{t_{0}<|t| \leq T} t^{1 / 2-(\eta-1)}|\zeta(3 / 2-\eta+i t) G(\eta+i t)| \frac{d t}{t^{m+1}} \\
& \ll k c(\eta)^{\omega(k)} x^{\eta}+k c(\eta)^{\omega(k)} x^{\eta} \int_{t_{0}<t \leq T} \frac{t^{3 / 2-\eta}}{t^{3}} d t . \\
& \ll k c(\eta)^{\omega(k)} x^{\eta} .
\end{aligned}
$$

Now we will estimate the contributions coming from the upper horizontal line (estimation for the lower horizontal line is similar).

The horizontal lines in total contribute a quantity which is in absolute value

$$
\begin{aligned}
& \ll \int_{\eta}^{c}\left|\zeta(\sigma-1+i T) G(\sigma+i T) \frac{x^{\sigma+i T}}{(\sigma+i T)(\sigma+1+i T) \cdots(\sigma+m+i T)}\right| d \sigma \\
& \ll c(\eta)^{\omega(k)} k\left(\int_{\eta}^{1}+\int_{1}^{3 / 2}+\int_{3 / 2}^{c}\right)|\zeta(\sigma-1+i T)| \frac{x^{\sigma}}{T^{k+1}} \\
& \ll k c(\eta)^{\omega(k)}\left\{\left(\int_{\eta}^{1 / 2}+\int_{1 / 2}^{1}+\int_{1}^{3 / 2}\right) T^{1 / 2-\sigma+1}|\zeta(2-\sigma+i T)| \frac{x^{\sigma}}{T^{m+1}} d \sigma\right. \\
& \left.\quad+\int_{3 / 2}^{c}|\zeta(\sigma-1+i T)| \frac{x^{\sigma}}{T^{m+1}} d \sigma\right\} \\
& \ll k c(\eta)^{\omega(k)}\left(\frac{x^{1 / 2}}{T^{m-1 / 2+\eta}}+\frac{x \log T}{T^{m}}+\frac{x^{3 / 2}(\log T)^{3 / 2}}{T^{m+5 / 6}}+\frac{x^{2}(\log T)^{3 / 2}}{T^{m+5 / 6}}\right)
\end{aligned}
$$

Collecting all the estimates, and taking $T=x^{10}$, for $m \geq 2$ we get:

$$
E_{m, k}(x) \ll k c(\eta)^{\omega(k)} x^{\eta} .
$$

This proves Theorem 1.1.

Remark 4.1. For $m \geq 2$ we may try to move the line of integration slightly left of vertical line 0 . On the line $\Re s=0$, the function $G(s)$ has simple poles at the points $s(\ell, p)=\frac{2 \pi i \ell}{\log p} \forall \ell \in \mathbb{Z}$ and for each prime $p \mid k$. let $p_{1}, p_{2}, \cdots p_{r_{k}}$ be the primes dividing $k$. The total contribution from the simple poles at the points $s(\ell, p)=\frac{2 \pi i \ell}{\log p_{j}}$ for $1 \leq j \leq r_{k}$ is given by:

$$
M=\sum_{j=1}^{r_{k}} \sum_{|\ell| \leq \frac{T \log p_{j}}{2 \pi}} \zeta\left(\frac{2 \pi i \ell}{\log p_{j}}-1\right) \prod_{p_{i} \neq p_{j}}\left(1-\frac{p_{i}}{p_{j}^{\frac{2 \pi i \ell}{\log p_{j}}}}\right) \frac{x^{\frac{2 \pi i \ell}{\log p_{j}}}}{\frac{2 \pi i \ell}{\log p_{j}}\left(\frac{2 \pi i \ell}{\log p_{j}}+1\right) \cdots\left(\frac{2 \pi i \ell}{\log p_{j}}+m\right)} .
$$

If one establishes that $M=o\left(x^{\eta}\right)$, then this will improve the error term. This seems to be really difficult. 
Remark 4.2. From the Theorem 1.1 observe that

$$
E_{1, k}(x) \ll_{\epsilon} x^{1 / 2+10 \epsilon}
$$

uniformly for $3 \leq k \ll x^{\epsilon}$ since $\omega(k) \ll \frac{\log }{\log \log k}$ for $k \geq 3$. Also $E_{m, k}(x) \ll x^{c_{1} \eta}$ uniformly for $3 \leq k \ll x^{\epsilon}$, where $c_{1}$ is effective positive constant.

\section{Acknowledgment.}

The author would like to thank Prof. S. D. Adhikari and Prof. A. Sankaranarayanan for suggesting the problem and for all fruitful discussion and suggestions.

\section{References}

[ABS] S. D. Adhikari, R. Balasubramanian and A. Sankaranarayanan, On an error term related to the greatest divisor of $n$ which is prime to $k$, Indian J. pure and appl. Math., 19(9) (1988), 830-841.

[AB] S. D. Adhikari and R. Balasubramanian, A note on a certain error term., Arch. Math., 56 (1991), $37-40$.

[AS] S. D. Adhikari and K. Soundararajan, Towards the exact nature of a certain error term-II , Arch. Math., 59 (1992), 442-449.

[In] A. E. Ingham, The distribution of prime numbers, Cambridge University Press (1995).

[Iv] A. Ivić, The Riemann Zeta-Function: Theory and Applications, Dover Publications, Inc, New York.

[JV] V. S. Joshi and A. M. Vaidya, Topics in Classical Number Theory, Colloq. Math. Soc. János Bolyái. Budapest (Hungary) 34 (1981).

[MH] T. Maxsein and J. Herzog, Mathematiches Forschungsinstitut oberwalfach Tagungsbericht, 42 (1986).

[SS] A. Sankaranarayanan and S.K. Singh, On the Riesz mean of $\frac{n}{\phi(n)}$, Hardy-Ramanujan Journal, 36 (2013), 8-20.

[T] E. C. Titchmarsh, The Theory of the Riemann Zeta function, (revised by D. R. Heath-Brown), Clarendon Press, Oxford (1986).

Saurabh Kumar Singh

Stat-Math Unit, Indian Statistical Institute

203 BT Road, Kolkata-700108, India.

e-mail: skumar.bhu12@gmail.com 